\title{
ANALYTIC CONTINUATION OF HOLOMORPHIC FUNC- TIONS WITH VALUES IN A LOCALLY CONVEX SPACE
}

\author{
WITOLD M. BOGDANOWICZ
}

Horváth [3] has announced a result generalizing the result of Gelfand and Shilov [2] on analytic continuation of holomorphic functions with values in a locally convex space. In this paper we shall present a generalization of these results which permits one to prove the existence of strong holomorphic extensions from the existence of weak or weak* holomorphic extensions. For more general results see [5].

1. Definition of a norming triple. Let $Y$ be a complex linear space and $p$ a seminorm on it. Assume that there is given a complete seminormed space $\left(Y_{p},\|\|_{p}\right)$ and a bilinear functional $(,)_{p}$ from the space $Y \times Y_{p}$ into the space $C$ of complex numbers. We shall say that the triple $\left(Y_{p},\|\|_{p},(,)_{p}\right)$ is a norming triple for the seminorm $p$ if

$$
p(y)=\sup \left\{\left|(y, z)_{p}\right|: z \in Y_{p},\|z\|_{p} \leqq 1\right\} \quad \text { for all } y \in Y .
$$

Example 1. Let $p$ be a seminorm on the space $Y$. Denote by $Y_{p}$ the family of all linear functionals $z$ on the space $Y$ such that $|z(y)|$ $\leqq c p(y)$ for all $y \in Y$ and some $c>0$. Define the norm of the functional by $\|z\|_{p}=\inf c$, where the infimum is taken over all constants satisfying the previous condition. Define the bilinear functional by means of the formula $(y, z)_{p}=z(y)$ for all $y \in Y, z \in Y_{p}$.

Using the generalization of the Hahn-Banach Theorem to the case of complex linear spaces one can easily prove that the triple $\left(Y_{p}\right.$, \|\|$\left._{p},(,)_{p}\right)$ is norming for the seminorm $p$.

EXAMPLE 2. Let $E$ be a sequentially complete, complex, locally convex space. Assume that the topology on it is generated by the family $Q=\{q\}$ of seminorms. Consider the strong dual $Y=E^{\prime}$. One may assume that the topology on the space is generated by the family of seminorms $p$ given by means of the formula:

$$
p(y)=\sup \{|y(z)|: z \in B\}
$$

for all $y \in Y$, where $B$ runs through all bounded sets $B$ of the space $E$, which can be represented in the form $B=\left\{z \in E: q(z) \leqq c_{q}\right.$ for all $q \in Q\}, c_{q}$ being a family of positive constants.

Define an extended seminorm by means of the formula

Received by the editors April 6, 1968. 
$\|z\|_{p}=\sup \left\{c_{q}^{-1} q(z): q \in Q\right\} \quad$ for $z \in E$. Let $Y_{p}=\left\{z \in E:\|z\|_{p}<\infty\right\}$. Notice that the space $\left(Y_{p},\|\|_{p}\right)$ is a complete seminormed space and $B=\left\{z \in Y_{p}:\|z\|_{p} \leqq 1\right\}$. Define the bilinear functional by means of the formula $(y, z)_{p}=y(z)$ for all $y \in Y, z \in Y_{p}$. It is easy to see that the triple $\left(Y_{p},\|\|_{p},(,)_{p}\right)$ is a norming triple for the seminorm $p$.

2. Definition of a holomorphic function. Let $D$ be a domain in the complex plane $C$. Let $Y$ be a sequentially complete, complex, locally convex Hausdorff space. Assume that $f$ is a function from the domain $D$ into the space $Y$. We shall say that the function $f$ is holomorphic if for every point $x_{0} \in D$ there exists a positive radius $r$ such that $f(x)=\sum_{n=0}^{\infty} a_{n}\left(x-x_{0}\right)^{n}$ if $\left|x-x_{0}\right|<r$, where $a_{n} \in Y$ and

$$
\left\{x \in C:\left|x-x_{0}\right|<r\right\} \subset D \text {. }
$$

Assume that $p$ is a seminorm on the linear space $Y$ and that $\left(Y_{p},\|\|_{p},(,)_{p}\right)$ is a norming triple for the seminorm. It is easy to prove from the definitions of a norming triple the inequality $\left|(y, z)_{p}\right| \leqq p(y)\|z\|_{p}$ for all $y \in Y, z \in Y_{p}$. This implies that the functional $(\cdot, z)_{p}$ is continuous and therefore the scalar function $h$ defined by $h(x)=(f(x), z)_{p}$ for $x \in D$ is holomorphic in the classical sense, if the function $f$ is holomorphic from the set $D$ into the locally convex space $Y$.

One can prove the usual formula for the coefficients in the Taylor expansion of the holomorphic function:

$$
a_{n}=f^{(n)}\left(x_{0}\right) / n ! \quad \text { for } n=0,1,2, \cdots .
$$

Assume that $D$ is an open domain and $D_{1}$ is another open domain such that $D \subset D_{1}$.

THEOREM. Let $Y$ be a complex sequentially complete locally convex Hausdorff space with topology generated by a family $P=\{p\}$ of seminorms. To every seminorm $p$ let correspond a norming triple ( $Y_{p}$, \|\|$\left._{p},(,)_{p}\right)$. Let $f$ be a holomorphic function from the domain $D$ into the space $Y$ and assume that for every seminorm $p$ and every $z \in Y_{p}$ the holomorphic function $x \mapsto(f(x), z)_{p}$ has an extension to a holomorphic function on the domain $D_{1}$. Then there exists a holomorphic function $f_{1}$ from the domain $D_{1}$ into $Y$ such that $f_{1}(x)=f(x)$ for all $x \in D$.

Proof. The proof of the theorem is based on the folllowing lemmas.

LEMma 1. Let $p$ be a fixed seminorm from the the family $P$. Let $f(x)=\sum_{n=0}^{\infty} a_{n}\left(x-x_{0}\right)^{n}$ if $\left|x-x_{0}\right|<r_{0}$. Assume that the function 
$x \mapsto(f(x), z)_{p}$ has a holomorphic extension onto a disc $\left|x-x_{0}\right|<r$, where $r_{0}<r$. Then $\lim \sup \left(p\left(a_{n}\right)\right)^{1 / n} r \leqq 1$.

Proof of Lemma 1 . Since $(\cdot, z)_{p}$ is a linear continuous functional for every $z$ in $Y_{p}$ and

$$
f(x)=\sum_{n=0}^{\infty} a_{n}\left(x-x_{0}\right)^{n} \quad \text { if }\left|x-x_{0}\right|<r_{0},
$$

we have

$$
(f(x), z)_{p}=\sum_{n=0}^{\infty}\left(a_{n}, z\right)_{p}\left(x-x_{0}\right)^{n} \quad \text { if }\left|x-x_{0}\right|<r_{0} .
$$

Since the function on the left side of the last equality has a holomorphic extension onto the circle of radius $r$, we get that the series $\sum_{n=0}^{\infty}\left(a_{n}, z\right)_{p}\left(x-x_{0}\right)^{n}$ is convergent at every point of the disc $\left|x-x_{0}\right|<r$.

Take any number $0<a<r$ and consider the sequence of sets

$$
F_{m}=\left\{z \in Y_{p}:\left|\left(a_{n}, z\right)_{p}\right| a^{n} \leqq m \text { for all } n\right\} .
$$

It is easy to prove that the sets $F_{m}$ are closed, circled, and contain the element zero. Moreover, we have the equality $Y_{p}=\bigcup_{m=1}^{\infty} F_{m}$. The space $\left(Y_{p},\|\|_{p}\right)$ being a complete seminormed space implies that there exists a positive radius $s$ and $a$ positive integer $m$ such that $z \in F_{m}$ if $\|z\|_{p} \leqq s$. This implies that there exists a positive constant $s(a)$ such that $\left|\left(a_{n}, z\right)_{p}\right| a^{n} \leqq s(a)\|z\|_{p}$ for $z \in Y_{p}, n=0,1, \cdots$. Taking the supremum over all elements $z$ such that $\|z\|_{p} \leqq 1$ we get $p\left(a_{n}\right) a^{n} \leqq s(a)$ for all $n$. Thus

$$
\lim \sup \left(p\left(a_{n}\right)\right)^{1 / n} a \leqq 1
$$

for all $a<r$. Passing to the limit in the last inequality when $a \rightarrow r$ we obtain the conclusion of Lemma 1.

LEMMA 2. Let $a_{n} \in Y$ and assume the series $\sum_{n=0}^{\infty} a_{n}\left(x-x_{0}\right)^{n}$ converges if $\left|x-x_{0}\right|<r_{0}$. If for every seminorm $p \in P$ and every $z \in Y_{p}$ the function $x \mapsto(f(x), z)_{p}$ has a holomorphic extension onto the disc $\left|x-x_{0}\right|$ $<r$, where $r_{0}<r$, then the series $\sum_{n=0}^{\infty} a_{n}\left(x-x_{0}\right)^{n}$ converges almost uniformly in the disc $\left|x-x_{0}\right|<r$, in the topology of the space $Y$ to a continuous function $f_{1}$.

Proof of Lemma 2. Take any point $x$ from the disc $\left|x-x_{0}\right|<r$.

It follows from Lemma 1 that the series $\sum p\left(a_{n}\right)\left|x-x_{0}\right|^{n}$ is convergent for every seminorm $p \in P$. This implies that the sequence $s_{n}(x)=\sum_{j=0}^{n} a_{j}\left(x-x_{0}\right)^{j}$ is Cauchy in the space $Y$. The space being 
sequentially complete, there exists an element $f_{1}(x) \in Y$ such that

$$
f_{1}(x)=\lim s_{n}(x)=\sum_{n=0}^{\infty} a_{n}\left(x-x_{0}\right)^{n} .
$$

Notice that in the disc $\left|x-x_{0}\right| \leqq a<r$ we have the uniform estimate

$$
p\left(f_{1}(x)-s_{n}(x)\right) \leqq \sum_{j>n} p\left(a_{j}\right) a^{j}
$$

This implies that the function $s_{n}$ converges almost uniformly to the function $f_{1}$. Since the functions $s_{n}$ are continuous we get the continuity of the function $f_{1}$.

LEMмa 3. Let $f$ be a continuous function from a domain $D$ into the space $Y$. If for every regular closed curve $C$ contained with its interior in $D$ the line integral $\int_{C} f(x) d x$ is zero, then the function $f$ is holomorphic in $D$.

The proof of Lemma 3 is obvious.

LEMMA 4. Let $f$ be a holomorphic function from a disc $\left|x-x_{0}\right|<r_{0}$, into the space $Y$. If for every seminorm $p \in P$ and every $z \in Y_{p}$ the function $x \mapsto(f(x), z)_{p}$ has a holomorphic extension onto the disc $\left|x-x_{0}\right|<r$, where $r_{0}<r$, then there exists a holomorphic function $f_{1}$ from the disc $\left|x-x_{0}\right|<r$ into the space $Y$ such that $f_{1}(x)=f(x)$ if $\left|x-x_{0}\right|<r_{0}$.

The proof of the lemma follows from Lemmas 1, 2, and 3.

Take a point $x_{0} \in D$ and a point $x \in D_{1}$. By a chain joining the points $x_{0}$ and $x$ we shall understand a collection of open discs $S_{0}, \cdots, S_{n}$ contained in the domain $D_{1}$ and such that the center of the disc $S_{0}$ is the point $x_{0}$ and $S_{0} \subset D$, the center of the disc $S_{j}$ is in the disc $S_{j-1}$ for all $j$, and $x \in S_{n}$.

Lемма 5. Let $f$ satisfy the assumptions of the theorem. Let $S_{0}, \cdots, S_{n}$ be a chain joining the point $x_{0} \in D$ with the point $x \in D_{1}$. Then $f$ has a holomorphic continuation along the chain. If $S_{0}^{1}, \cdots, S_{m}^{1}$ is another chain joining the points $x_{0}$ and $x$ then the extension of the function $f$ along the first chain yields the same function on $S_{n} \cap S_{m}^{1}$ as the extension along the second chain.

Proof of Lemma 5. Denote by $g_{1}$ the holomorphic function on $S_{n}$ being the holomorphic extension of the function $f$ along the first chain. Let $g_{2}$ be the holomorphic function on the disc $S_{m}^{1}$ being the holomorphic extension of the function along the second chain. Since the scalar function $x \mapsto(f(x), z)_{p}$ has a unique extension from the do- 
main $D$ onto the domain $D_{1}$, we get $\left(g_{1}(x), z\right)_{p}=\left(g_{2}(x), z\right)_{p}$ if $x \in S_{n} \cap S_{m}^{1}$. The element $z \in Y_{p}$ being arbitrary, we get $p\left(g_{1}(x)-g_{2}(x)\right)=0$ for all $p \in P$. Since the space $Y$ is Hausdorff, we conclude $g_{1}(x)=g_{2}(x)$ on $S_{n} \cap S_{m}^{1}$.

Proof of the Theorem. Take any point $x \in D_{1}$ and consider a fixed point $x_{0} \in D$. Define a function $f_{1}$ by means of the formula $f_{1}(t)=g(t)$ for all $t \in S$, where $g$ represents a holomorphic function on the disc, $S_{n}=S$ being a holomorphic extension along the chain $S_{0}, \cdots, S_{n}$ joining the points $x_{0}, x$. It follows from Lemma 5 that the function $f_{1}$ is well defined. Moreover the function represents a holomorphic extension of the function $f$ from the domain $D$ onto the domain $D_{1}$.

The following corollary represents a generalization of the result due to Horváth [3]. We have removed the assumption that the space $Z$ is Hausdorff.

Corollary 1. Let $Z$ be a sequentially complete, barreled, complex, locally convex space and let $Y=Z^{\prime}$ be the strong dual, i.e. with topology of uniform convergence on all bounded sets of $Z$. Let $f: D \rightarrow Y$ be a holomorphic function such that each of the scalar functions $x \mapsto f(x)(z)$, $z \in Z$, has a holomorphic extension onto the set $D_{1}$. Then there exists a holomorphic function $f_{1}: D_{1} \rightarrow Y$ extending the function $f$.

The proof of the corollary follows immediately from Example 2. Indeed notice that the topology on the space $Y$ can be introduced by means of the seminorms

$$
p_{c}(y)=\sup \left\{|y(z)|:\|z\|_{c} \leqq 1\right\}
$$

where

$$
\|z\|_{c}=\sup \left\{c_{q}^{-1} q(z): q \in Q\right\} \quad \text { for } z \in Z,
$$

and $c$ denotes any function $q \mapsto c_{q}>0$ for all $q \in Q$. The space $Y$ endowed with the family of seminorms $\left\{p_{c}\right\}$ is a complete, Hausdorff, complex, locally convex space [4]. Notice that the triple $\left(Y_{c},\|\|_{c}\right.$, $(,)_{c}$ ) is norming for the functional $p_{c}$ where $Y_{c}=\left\{z \in Z:\|z\|_{c}<\infty\right\}$, and $(y, z)_{c}=y(z)$ for all $y \in Y, z \in Y_{c}$ according to Example 2. Thus all the assumptions of the theorem are satisfied.

CoROllaRY 2. Let $Z$ be a complete seminormed space and let $Y=Z^{\prime}$ be its strong dual. Let $f$ be a holomorphic function from $D$ into $Y$ such that for every $z \in Z$ the scalar function $x \mapsto f(x)(z)$ has a holomorphic extension onto the set $D_{1}$. Then there exists a holomorphic function $f_{1}$ from $D_{1}$ into $Y$ extending the function $f$. 
Notice that this corollary is a particular case of Corollary 1.

Corollary 3. Let $Y$ be a sequentially complete, Hausdorff, complex, locally convex space. Let $f: D \mapsto Y$ be a holomorphic function such that for every linear continuous functional $y^{\prime} \in Y^{\prime}$ the scalar function $x \mapsto y^{\prime} f(x)$ has a holomorphic extension onto the set $D_{1}$. Then there exists a holomorphic function $f_{1}: D_{1} \mapsto Y$ extending the function $f$.

The proof of the corollary follows from Example 1.

Corollary 4. Let $Y$ be a complex Banach space. Let $f$ be a hoiomorphic function from the domain $D$ into the space $Y$ such that for every linear continuous functional $y^{\prime} \in Y^{\prime}$ the scalar function $x \mapsto y^{\prime} f(x)$ has a holomorphic extension onto the domain $D_{1}$. Then there exists a holomorphic function $f_{1}: D_{1} \mapsto Y$ extending the function $f$.

This corollary is a particular case of the preceding one.

Corollary 4 represents a generalization of the result due to Horváth [3, Theorem 2], proven for the case of the space $Y=c_{0}$ of sequences convergent to zero.

Compare the following with [6], [7].

Let $Q$ be a compact set in the space $R^{n}$. Assume that the interior of the set $Q$ is dense in $Q$. Let $C(Q), L(Q), H(Q, D)$ be, respectively, the space of complex continuous functions on $Q$, the space of Lebesgue summable functions on $Q$, the space of all continuous functions $f$ from $Q \times D$ into the space $C$ of complex numbers such that for every $q \in Q$ the function $f(q, \cdot)$ is holomorphic.

CoROllaRY 5. Let $f \in H(Q, D)$ and assume that for every function $g \in L(Q)$ the function $\int g(q) f(q, \cdot) d q$ has a holomorphic extension onto the set $D_{1}$. Then there exists a function $f_{1} \in H\left(Q, D_{1}\right)$ extending the function $f$.

Notice that the space $H(Q, D)$ can be considered as the space of holomorphic functions from $D$ into $C(Q)$ and that the triple $\left(L(Q),\|\|_{L},(, \quad)_{L}\right)$, where $\|f\|_{L}=\int|f(q)| d q$ for all $f \in L(Q)$, and $(f, g)_{L}=\int f(q) g(q) d q$ for all $g \in L(Q)$ and $f \in C(Q)$, is norming for the norm in the space $C(Q)$. This follows from [1, Theorem 5, p. 289].

\section{REFERENCES}

1. N. Dunford, and J. Schwartz, Linear operators. I: General theory, Pure and Appl. Math vol. 7, Interscience, New York, 1958.

2. I. M. Gelfand and G. E. Shilov, Generalized functions. Vol. I: Operations on them, Chapter I, Appendix 2, No. 3., Fizmatgiz, Moscow, 1958; English transl., Academic Press, New York, 1964. 
3. John Horvath, The analytic continuation of vector-valued holomorphic functions, Notices Amer. Math. Soc. 15 (1968), p. 176.

4. - Topological vector spaces and distributions, Addison-Wesley, Reading, Mass., 1966.

5. W. M. Bogdanowicz, Analytic continuation of locally-convex-space-valued holomorphic functions on domains in real or complex locally convex spaces, Math. Ann. (to appear). For the announcement of the main result see Proceedings of the Symposium in Functional Analysis, September 29-October 5, 1968, Oberwolfach, West Germany.

6. - On analytic extensions of holomorphic functions with values in the space of continuous functions, Notices Amer. Math. Soc. 15 (1968), p. 627.

7. - Existence of analytic extensions of holomorphic functions with values in the space of Lebesgue summable functions, Notices Amer. Math. Soc. 15 (1968), p. 792.

Catholic University of America 